\title{
Exact and efficient isogeometric reanalysis of accurate shape and boundary modifications
}

\author{
Chensen Ding ${ }^{1,2,3}$, Xiangyang Cui ${ }^{1,2^{*}}$, Guanxin Huang ${ }^{1,2}$, Guangyao $\mathrm{Li}^{1,2}$, Kumar. K. Tamma ${ }^{3}$ \\ ${ }^{1}$ State Key Laboratory of Advanced Design and Manufacturing for Vehicle Body, Hunan University, \\ Changsha 410082, P. R. China \\ ${ }^{2}$ Collaborative Innovation Center of Intelligent New Energy Vehicle, Shanghai 200092, P. R. China \\ ${ }^{3}$ Department of Mechanical Engineering, University of Minnesota-Twin Cities, Minneapolis, MN \\ 55455, United States
}

\begin{abstract}
In the traditional design-through-analysis pipeline, geometric models and modifications are approximately represented and transformed with computational models. Besides, after each modification, the latest design often needs to be completely analyzed again leading to reanalysis. These procedures produce many errors and are extremely time-consuming. Therefore, in this paper, we propose a novel, and an exact and efficient isogeometric reanalysis methodology of accurate shape and boundary modifications that improves the totality of integration of design and analysis greatly. Geometric models and shape modifications are exactly represented and transformed. And, the corresponding computational models will change simultaneously when the geometric models are modified; thereby, reducing error and time in model representation and transformation. Furthermore, we extend and propose the isogeometric based exact reanalysis method termed Indirect Factorization Updating (IFU) with the combination of isogeometric based reanalysis. The method can efficiently obtain the exact solution of the modified structure, without solving the complete set of modified equations of the new structure. It is also applicable to all techniques for representing the CAD geometry model and complex problems. Several examples illustrate and verify the accuracy and efficiency of this proposed method; and furthermore, the larger the scale of the problem, the more advantageous the end result will be.
\end{abstract}

Key words: Isogeometric Analysis (IGA); Exact Reanalysis; Indirect Factorization Updating (IFU); Shape and Boundary Modifications

* Corresponding author: X. Y. Cui. Tel: +86-073188821717. E-mail: cuixy@hnu.edu.cn 


\section{Introduction}

The traditional, and still dominant, technique of realizing analysis and design is depicted in Fig. 1(a). It mainly includes computer-aided design (CAD) model representation, modification and computer-aided engineering (CAE) analysis. In each design process, the engineer firstly generates the CAD model and translates it into an an analysis-suitable geometry. Next, the geometry is meshed and analyzed such as via the finite element analysis (FEA). According to the analysis results, the original CAD model is therein often modified or optimized [1-3]. The entire design and analysis procedure includes two crucial ingredients that are also the bottlenecks in traditional CAD/CAE integration. The first one is how to accurately, simply and freely represent the exact geometry and adjust the design (model). The second one is how to exactly and quickly get the analysis results of the modified structure; for which, the analysis process will repeat several times until the modified structure meets the design requirements.

Considering the first bottleneck via traditional techniques, the question is how to accurately, simply and freely represent the exact geometry and adjust the design (model). Due to the fact that models represented and used in CAD and CAE are totally different, there is a huge gap between CAD and CAE in traditional design and analysis [4-7]. Firstly, the model in the CAD field is generally represented by splines, such as NURBS (Non-Uniform Rational B-splines), and it is continuous and parameterized. While, the model represented and used in the CAE field is an approximation and a discrete model of the exact geometry. Consequently, the model used in CAE analysis must be newly regenerated from the CAD geometry. Furthermore, the CAE analysis suitable models cannot be easily and exactly created from the CAD geometry, especially for complex domains. This generation is much more involved than simply generating a mesh. There are many time consuming, preparatory steps involved such as geometry clean-up, feature removal and so on [7]. Even when only considering meshing or geometry updates, due to the fact that the modification is decided and made in the CAE model according to the CAE analysis result, this modification must be transformed subsequently into the CAD geometric model. And the modified CAD model should be subsequently converted, such as remeshing, into the CAE model to reanalyze again. Both of these two transformations produce and accumulate a lot of error in the geometry representation and transformation.

Regarding the second issue mentioned above, the question is how to exactly and 
efficiently obtain the corresponding CAE result of the modified structure. Due to the fact that almost all of the structural responses cannot be obtained readily by experiment or analytical methods, various numerical methods, for example FEM, are widely used. Using numerical methods implies solving a set of simultaneous equations; this requires huge computational resources, such as computer memory and computational times, especially for large-scale problems. Even for a small modification produced by the designer, the set of simultaneous equations is newly produced and needs to be completely solved again to obtain the CAE result of the modified structure. Furthermore, this CAE analysis procedure will repeat several hundreds of times in design or optimization, costing enormous computational time and making the whole design loop and analysis very inefficient.

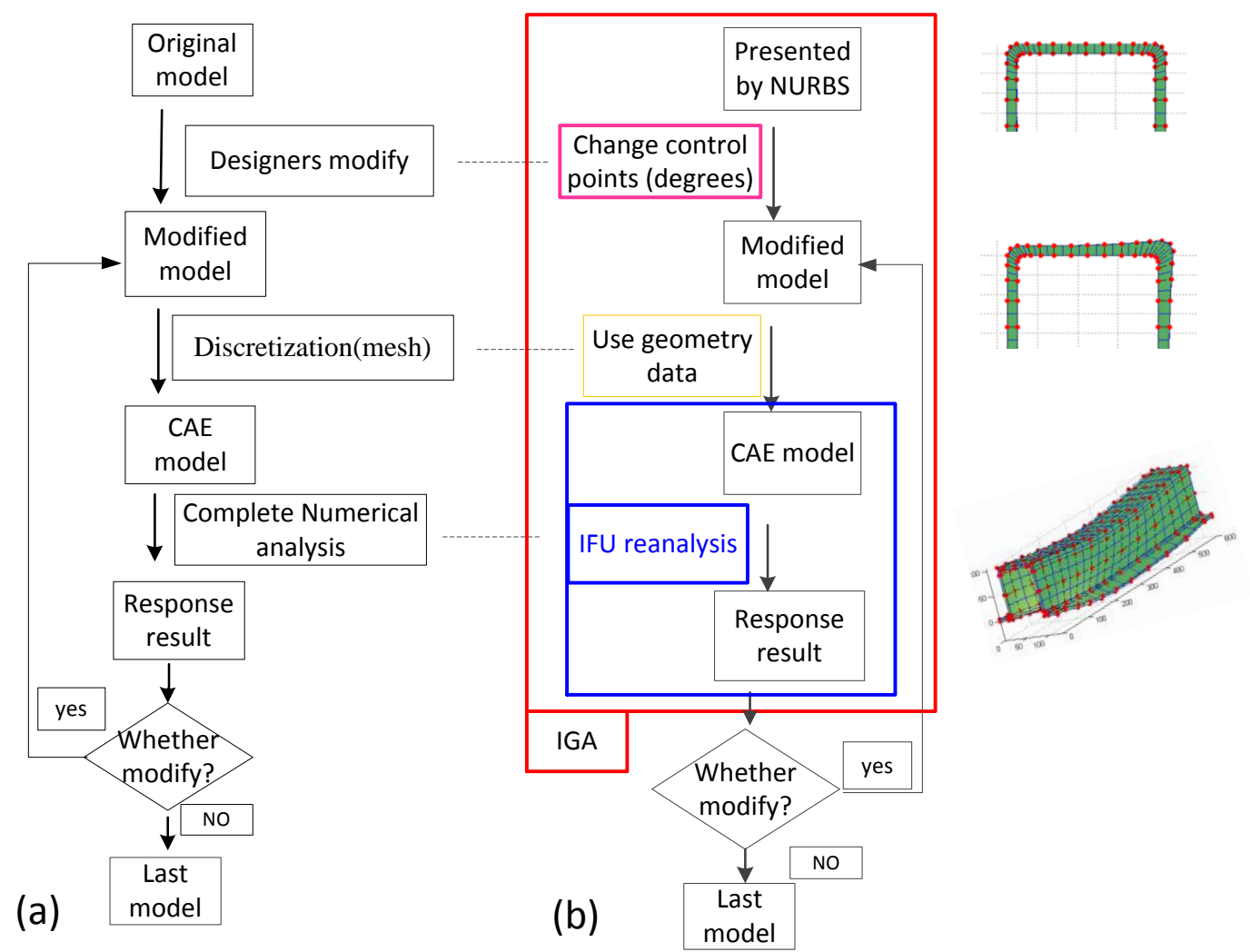

Fig. 1. Architectures of traditional (a) and proposed (b) design and analysis.

Therefore, in order to resolve the two critical bottlenecks existing in traditional methods, this paper proposes an exact and efficient isogeometric reanalysis of accurate local modifications in shape and boundary based upon isogeometric analysis and reanalysis, whose architecture is shown in Figure 1(b) and also compared with FEM based design and analysis. To resolve the first issue, we adopt the IGA method that was proposed and enriched by Hughes $[6,7]$ to build a tighter connection between $\mathrm{CAD}$ and CAE. We can exactly represent the geometric model by using 
splines, such as non-uniform rational B-splines (NURBS), and employ the same spline basis functions as interpolation functions to approximate the solution field. In this manner, we eliminate the error of the geometry model representation and can integrate $\mathrm{CAD}$ and $\mathrm{CAE}$. Meanwhile, the analysis accuracy is improved due to the spline basis functions having higher continuity. Furthermore, the corresponding CAE analysis model will change at the same time as that of the geometric CAD model modification. Therefore, no transformation between the CAD model and the CAE model is needed anymore; thereby reducing significant errors and computational times.

In addition, compared to other numerical methods, isogeometric analysis generally has several major advantages as follows. First, IGA has a great flexibility and precision in the definition of the geometry and can preserve the original model in the entire process. Second, when the expected accuracy level is the same, significantly less number of system equations are needed and less computational cost is required; this makes IGA to have a good efficiency and applicability. Third, it is easier to refine the mesh than FEM and has greater accuracy in satisfaction of the essential boundary conditions. Due to these features, IGA has attracted many researchers to work in its theory and various engineering applications, such as shape and topology optimization [9-14], fluid mechanics [15-17] and fluid-structure interaction problems [18-20], contact mechanics [21-23], and damage and fracture mechanics [24-26] and the like.

In order to resolve the second problem regarding iterative CAE analysis, a novel reanalysis concept is introduced, and the IGA based exact reanalysis method - IFU (Indirect Factorization Updating) is proposed to efficiently and exactly analyze modified structures with shape and boundary modifications. For reanalysis techniques, they are initially proposed to analyze the modified structure without completely solving the new set of modified analysis equations; thereby, avoiding the high CAE computational cost in iterations. After recent developments, many accomplishments about reanalysis method have been obtained and they also have been successfully employed in various classes of structural reanalysis problems, such as optimization, vibration and nonlinear problems. Generally, reanalysis methods, from the aspects of the accuracy and modified scale, can be mainly classified into two categories: approximate methods and Direct Methods (DMs). Approximate methods can deal with high-rank or global modification, and an exact solution usually cannot be obtained by using them. Among approximate methods, the Combined Approximation (CA) method [27-30] proposed by Kirsch [27] appears to be the most popular one in recent years. The advantage of $\mathrm{CA}$ is the efficiency of local approximations and the 
accuracy of global approximations. Also, it has been extended to multidisciplinary fields, such as eigenvalue-problem, nonlinear analysis and dynamic reanalysis [31-35], etc. Based upon CA, Chen suggested a universal Iterative Combined Approximation (ICA) approach and various types of topological modifications [36]. Moreover, there exist many other approximate reanalysis methods that have been well developed [3745]. For example, Kirsch introduced a classical iterative algorithm-Preconditioned Conjugate Gradient (PCG) for structural reanalysis [39], and Wu developed the PCG method considering both removing and adding Degrees of Freedom (DOFs) [40-42]. Yang proposed a matrix perturbation method for structural modal reanalysis [43, 44]. Based upon rational approximation, $\mathrm{Wu}$ developed a reanalysis method that can have very large changes in the design variables [45].

Compared with approximate methods, DMs generally can give exact solutions of modified structures and are suitable for low-rank or local modification. The first DM proposed by Sherman and Morrison [46] gave an exact solution where the change was only a given column or row of the original matrix. Later, Woodbury [47] developed the Sherman-Morrison-Woodbury (SMW) formula to a situation where a relatively small proportion is modified. The SMW formula is an exact reanalysis method and efficient to deal with low-rank modifications. As Tuckerman [48] stated, any DM is an explicit or implicit application of the SMW formula, and it could give a foundation for further research of DMs. However, the SMW formula is an implicit call of the initial analysis information; which means if multiple modifications or working conditions need to be analyzed, the SMW formula should be carried out repeatedly, and the computational cost will dramatically increase. Therefore, there is another kind of widely used DMs that are based on matrix factorization updating. The advantage of factorization updating is that the updated factorization can be reused even when the modification or working condition is multiple. Davis developed a sparse technique to obtain the new factorization for rank-one modifications [49] and developed it for multiple-rank modifications [50]. Liu advanced Davis's work for structural reanalysis with added DOFs [51] and supports modifications [52]. Song proposed a direct reanalysis algorithm to update the triangular factorization in sparse matrix solution [53]. Moreover, there are also many other DMs under development. For example, Cheikh suggested a DM based on a Moore Penrose generalized inverse for static reanalysis of structures [54]. The author Huang proposed the Independent Coefficients (IC) method for large-scale problems [55] and FEM based IFU for boundary modification [56].

Besides, based upon the scale of the modified DOFs that is crucial to every 
reanalysis method, they can be classified into three categories: small, medium and large. Among all these three categories, we found several works that mentioned the details of the changes, such as the fixed DOFs with total DOFs, and highlight them in Table 1 [57]. For example, reanalysis methods for small change [46, 47, 58, 59] represented change details that they handle. And for medium scales, the works [60, 61, $62,55]$ also mentioned how and to what extent the structures were modified, as well the updated triangular factorization algorithm [53] to handle large changes.

Table 1. Reanalysis methods with different scales of modification.

\begin{tabular}{lll}
\hline Changes & Methods & Modified DOFs/initial DOFs \\
\hline Small & Sherman-Morrison formula [46] & 1-rank change \\
& SMW formula [47] & m-rank changes (m small value) \\
& The theorems of geometric variation [58, & Geometric variation, 18.75\% (144nodes) \\
& 59] & \\
Medium & Sub-structuring technique [60] & 99/2973 (cross-section area) \\
\cline { 2 - 3 } & Equilibrium equations and SVD [61] & Modified 386 DOFs/1186 DOFs \\
& Using graph products [62] & Irregular 924 DOFs / Regular 18480 \\
& & DOFs \\
& Independent coefficients [55] & Modified part <5\% (Initial DOFS \\
& & $\left.1.7 * 10^{6}\right)$ \\
Large & An updated triangular factorization & $5.38 \%$ (Initial DOFs 442331), 1.11\% \\
& algorithm [53] & $($ Initial DOFs 1285044) \\
\hline
\end{tabular}

In this work, we advance and extend and propose the IGA exact reanalysis method termed Indirect Factorization Updating (IFU) for shape and boundary modifications based upon the IC and the SMW formula. This method can represent the geometric model and shape modification exactly; the corresponding CAE analysis model will change simultaneously when the CAD geometric model is modified. Meanwhile, the IFU method can efficiently obtain the exact CAE result of the latest structures with shape and boundary modifications, and the resulting accuracy is preserved and guaranteed no matter how many DOFs are modified, and/or how to modify them. Furthermore, the larger the scale of the problem, the more advantageous the proposed method will be. This method takes full use of the advantages of IGA in $\mathrm{CAD} / \mathrm{CAE}$ integration and IFU in providing exact and efficient CAE reanalysis; and it also provides a powerful tool for future researchers in solving problems that are largescale or need iterations, as well as being extremely valuable in real world engineering problems.

The remainder of this paper is organized as follows. We briefly introduce isogeometric analysis in Section 2. Section 3 will present the reanalysis problems and proposed IFU. In Section 4, two examples are presented to verify the effectiveness 
and efficiency of IGA based IFU reanalysis for shape and boundary modifications. Finally, some conclusions are drawn in Section 5.

\section{Brief highlights on Isogeometric Analysis (IGA)}

The concept of Isogeometric Analysis was firstly proposed by Hughes [6]. It greatly improved the process of integrating finite element analysis (FEA) analysis and CAD design. The biggest difference between this method and other numerical methods is that the spline basis functions, such as B-splines or NURBS, are used to construct the exact geometric model and are employed as interpolation functions in CAE analysis. The problem domain is discretized by the existing control points defining CAD geometry rather than using approximating meshes as in finite elements, finite difference grids or collection of points as in the meshfree methods; thereby, it eliminates the error in representing geometrical models and reduces errors associated with approximating solution fields because of higher continuity of the basis functions.

\subsection{CAD used in IGA}

The spline basis functions are the key to IGA, and they are used to construct the geometry and used as interpolation functions in CAE analysis at the same time. Hence, this section which is based on Piegl's work [8] provides a short recapitulation of the often used splines: Non-Uniform Rational B-splines (NURBS).

Generally, a $p$ th degree NURBS curve is defined by

$$
\mathbf{C}(\xi)=\frac{\sum_{i=1}^{n} B_{i, p}(\xi) \omega_{i} \mathbf{P}_{i}}{\sum_{j=1}^{n} B_{j, p}(\xi) \omega_{j}}=\sum_{i=1}^{n} N_{i, p}(\xi) \mathbf{P}_{i} \quad 0 \leq \xi \leq 1
$$

where $\mathbf{P}_{i}$ represent the coordinate positions of a set of $i=1,2,3, \ldots n$ control points, $w_{i}$ are the corresponding weights, $B_{i, p}(\xi)$ and $N_{i, p}(\xi)$ represent the $p$ th degree Bspline and NURBS basis functions defined on the given knot vector $\Xi=\left\{\xi_{1}, \xi_{2}, \ldots, \xi_{m}\right\}$, the number of knots $m=n+p+1$. As an example, Fig. 2 shows all non-zeros cubic NURBS basis functions for an open knot vector $\Xi=\{0,0,0,0,0.25,0.5,0.75,1,1,1,1\}$. 
The $p$ th $(i=1,2,3, \ldots n)$ B-spline basis function $B_{i, p}(\xi)$ can be defined recursively as,

$$
\begin{aligned}
& B_{i, p}(\xi)=\frac{\left(\xi-\xi_{i}\right) B_{i, p-1}(\xi)}{\xi_{i+p}-\xi_{i}}+\frac{\left(\xi_{i+p+1}-\xi\right) B_{i+1, p-1}(\xi)}{\xi_{i+p+1}-\xi_{i+1}} \\
& B_{i, 0}(\xi)= \begin{cases}1 & \xi_{i} \leq \xi \leq \xi_{i+1} \\
0 & \text { Otherwise }\end{cases}
\end{aligned}
$$

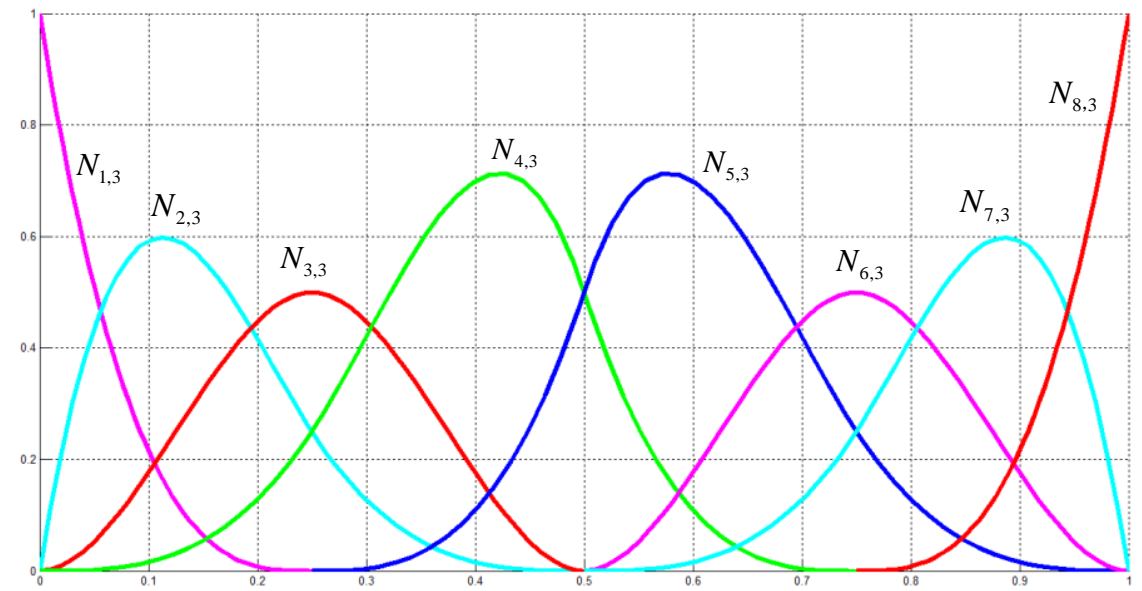

Fig. 2. Cubic basis functions for an open knot vector $\Xi=\{0,0,0,0,0.25,0.5,0.75,1,1,1,1\}$

For 3-D problems, based upon the basis function $B_{i, p}(\xi)$ and given knot vectors $\Xi_{1}=\left\{\xi_{1}, \xi_{2}, \ldots, \xi_{m}\right\} \quad \Xi_{2}=\left\{\eta_{1}, \eta_{2}, \ldots, \eta_{m}\right\} \quad \Xi_{3}=\left\{\zeta_{1}, \zeta_{2}, \ldots, \zeta_{m}\right\}$, the solid element is the tensor product of the curves in three dimensions constructed by:

$$
V(\xi, \eta, \zeta)=\frac{\sum_{i=1}^{n_{1}} \sum_{j=1}^{n_{2}} \sum_{k=1}^{n_{3}} B_{i, p}(\xi) B_{j, q}(\eta) B_{k, r}(\zeta) w_{i, j, k} \mathbf{P}_{i, j, k}}{\sum_{l=1}^{n_{1}} \sum_{m=1}^{n_{2}} \sum_{n=1}^{n_{3}} B_{l, p}(\xi) B_{m, q}(\eta) B_{n, r}(\zeta) w_{l, m, n}}
$$

where $P_{i, j, k}$ are $n_{1} \times n_{2} \times n_{3}$ control points, $w_{i, j, \mathrm{k}}$ are the associated weights respectively; and $B_{i, p}(\xi), B_{j, q}(\eta), B_{k, r}(\zeta)$ are NURBS basis functions mentioned above.

\subsection{Spaces, integration and CAE formulations in IGA}

The spaces in FEM are shown in Fig. 3, and there are two types of spaces: parent space and physical space. There is only one type of mesh in the physical space named as the physical mesh. And there are two types of elements: physical element in physical space and parent element in parent space. The physical element is defined by its nodal physical coordinates, and the degrees of freedom are usually the values of 
the basis functions at the nodes. The basis functions are typically interpolatory and may take on positive and negative values. Additionally, integration is conducted in the parent element that is connected with physical element through isoparametric transformation.

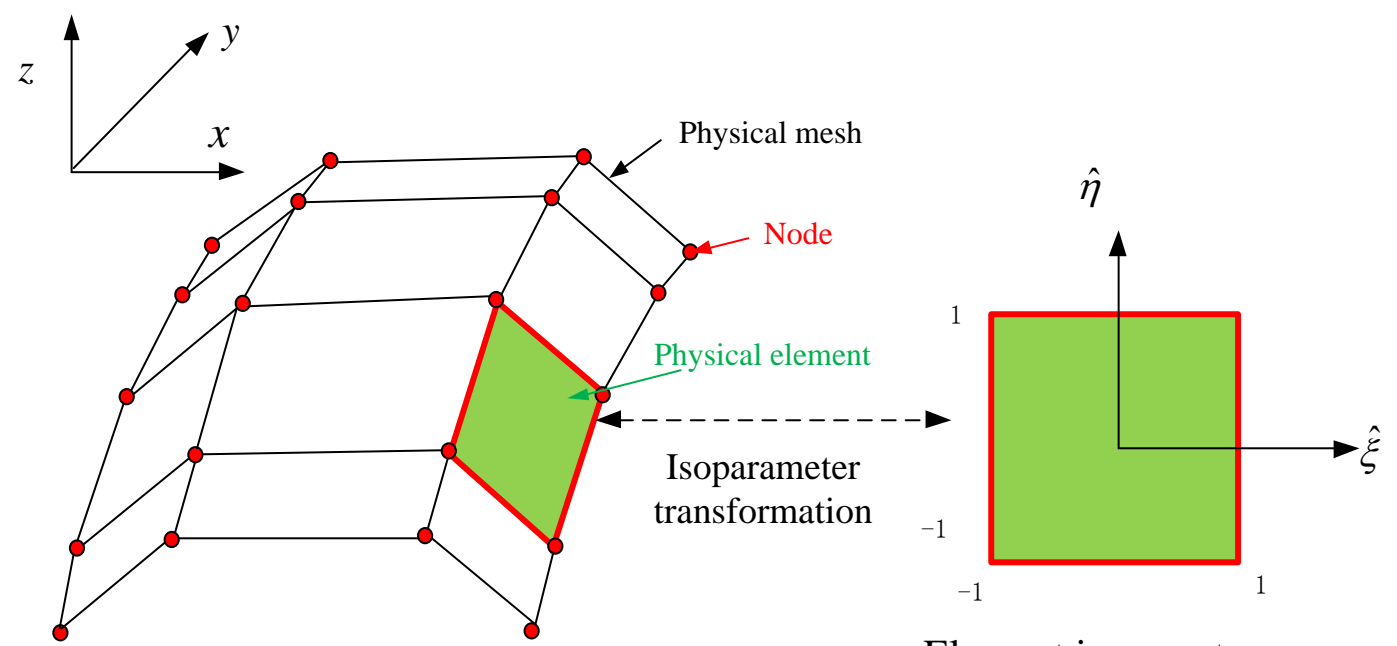

Physical space

Element in parent space

Fig. 3. Schematic illustration of spaces in FEM.

In IGA as is shown in Fig. 4, there are three types of spaces: the physical space, the parent space and the parameter space. And there two notions of mesh: the control mesh and the physical mesh. The control mesh is constructed by the control points and generally does not conform to the actual geometry. It appears like a typical finite element mesh of multi-linear elements and the degrees of freedom are located at the control points. We can simply and freely modify the structural shape simply by changing the control point positions. Meanwhile, once the shape is modified by changing control point positions, the CAE will change immediately, and no transformation procedure between the CAD model and CAE model is needed; thereby avoiding huge errors and computational cost. This is its unique advantage in structural design and optimization, and is also the key aspect of this paper to handle the first bottleneck mentioned before. The physical mesh is a decomposition of the actual geometry, and it is defined by the control mesh and knot vectors that form the parameter space. Additionally, an "element" in IGA is generally defined as the span between two distinct knot values in the parameter space. The number of elements in a curve is the number of non-zero knot spans in the knot vector. The integration, such as Gauss integration, is performed on the parent space that is connected to the parameter space by isoparametric transformation. 


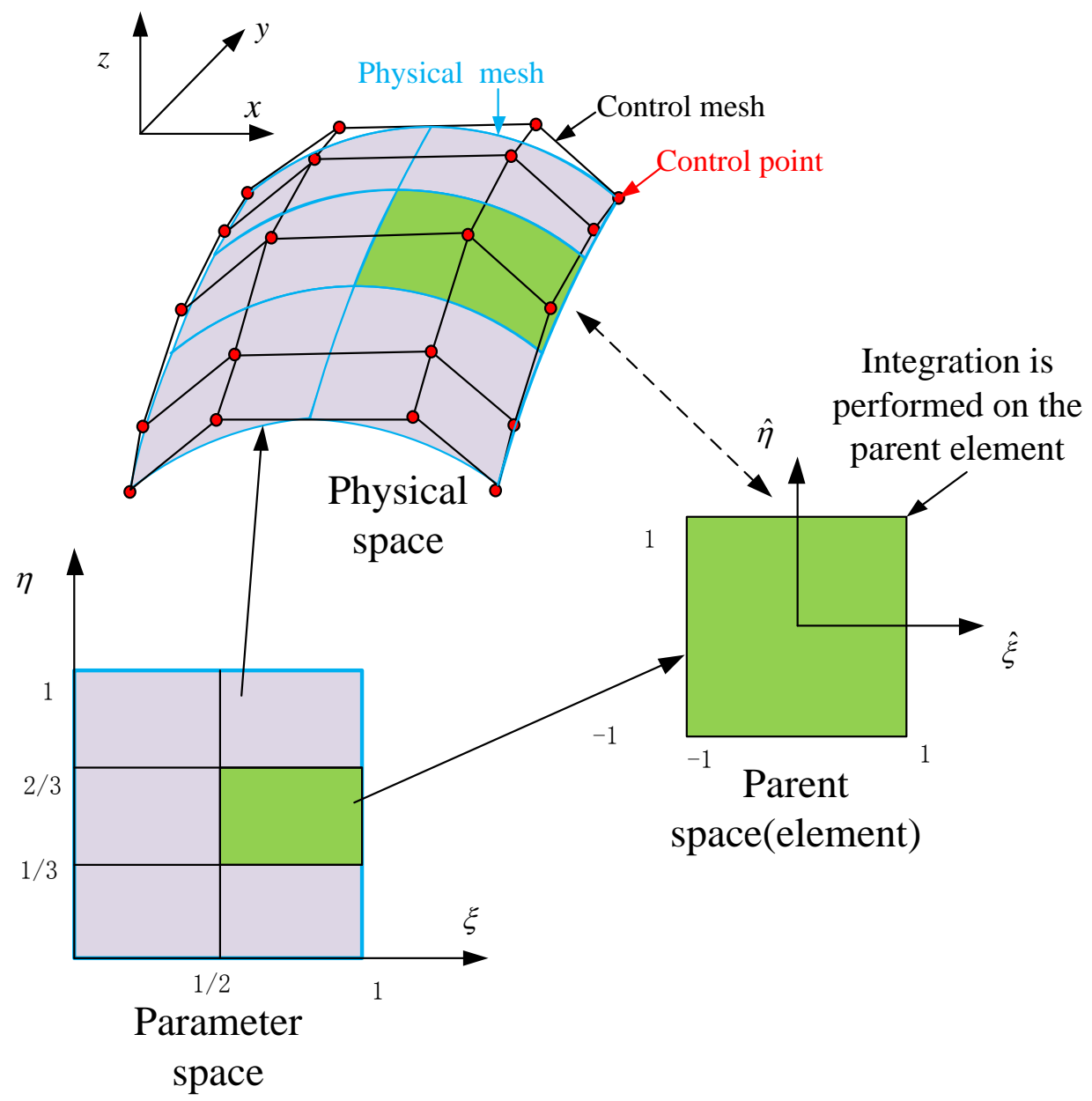

Fig. 4. Schematic illustration of space and integration in IGA.

For CAE simulations, most of the formulations, such as continuum mechanics formulations, in IGA are similar to formulations in FEM. Hence, in order to be concise and focus on the most important issue of this paper, we do not provide their details and can be found in $[4,9]$. However, the difference is that IGA uses the shape function that is used to construct the exact geometry as the interpolation function to approximate the unknown field in CAE analysis. Hence, the approximated displacement functions are written as:

$$
\begin{gathered}
\mathbf{u}(\xi, \eta, \zeta)=\sum_{i=0}^{n_{1}} \sum_{j=0}^{n_{2}} \sum_{k=0}^{n_{3}} R_{i, j, k}(\xi, \eta, \zeta) \mathbf{d} \\
\delta \mathbf{u}=\sum_{i=0}^{n_{1}} \sum_{j=0}^{n_{2}} \sum_{k=0}^{n_{3}} R_{i, j, k}(\xi, \eta, \zeta) \delta \mathbf{d}
\end{gathered}
$$

where $\mathbf{d}_{i}$ is the nodal displacement vector and $R_{i, j, k}(\xi, \eta, \zeta)$ is the NURBS basis function. 


\section{The reanalysis problem and IFU used in IGA}

\subsection{Basic theories of reanalysis and IC method}

As noted earlier, reanalysis methods are proposed to efficiently analyze structures that are modified due to some changes in the structure properties. Its objective is to use the result of the original structure to evaluate the modified structural result for such changes without solving the complete set of modified analysis equations; therein avoiding the high computational cost in iterations. The reanalysis problem can be stated as follows:

Assuming the initial equilibrium equations discretized by IGA of the initial structure are

$$
\mathbf{K}_{0} \mathbf{d}_{0}=\mathbf{F}
$$

where $\mathbf{d}_{0}$ are initial displacements. After modifications, the new equilibrium equations are

$$
\mathbf{K d}=\mathbf{F}
$$

For the Independent coefficients (IC) method [55], it replaces solving Eq. (6) directly with re-calculating the displacements of the DOFs influenced by the corresponding modifications.

Hence, assuming that the solution of the modified equations is

$$
\mathbf{d}=\mathbf{d}_{0}+\Delta \mathbf{d}
$$

Eq. (6) can be rewritten as

$$
\mathbf{K}\left(\mathbf{d}_{\mathbf{0}}+\Delta \mathbf{d}\right)=\mathbf{F}
$$

Transforming Eq. (8) into

$$
\mathbf{K} \Delta \mathbf{d}=\mathbf{F}-\mathbf{K} \mathbf{d}_{0}
$$

and defining

$$
\boldsymbol{\delta}=\mathbf{F}-\mathbf{K d}_{0}
$$

Eq. (9) becomes,

$$
\mathbf{K} \Delta \mathbf{d}=\boldsymbol{\delta}
$$

$\boldsymbol{\delta}$ can be regarded as the residual value of the initial solution $\mathbf{d}_{0}$. Since the modification is local, although there is no exact definition of "local", in this paper, we assume the modification is local when the percentage of modified DOFs to total DOFs is less than 10\%; only some members of $\boldsymbol{\delta}$ are non-zero. Pre-select a small tolerance $\varepsilon$, and all the DOFs related to the non-zero members of $\boldsymbol{\delta}$ should be recorded 
for the rest of the steps for the IC method. The selected DOFs are recorded in $\mathbf{S}_{d}$, and the number of the selected DOFs is $n_{d}$.

Assuming that

$$
\Delta \mathbf{d}=\mathbf{v}_{1} y_{1}+\mathbf{v}_{2} y_{2}+\cdots+\mathbf{v}_{s} y_{s}=\mathbf{r}_{B} \mathbf{y}
$$

where $\mathbf{v}_{1}, \mathbf{v}_{2}, \ldots, \mathbf{v}_{\mathrm{s}}$ are the basis vectors, and $y_{1}, y_{2}, \ldots, y_{\mathrm{s}}$ are the coefficients of every basis vector. Note that $s$ is the number of the basis vectors and equals to $n_{\mathrm{d}}$. In Eq. (12),

$$
\begin{aligned}
& \mathbf{r}_{B}=\left[\begin{array}{llll}
\mathbf{v}_{1} & \mathbf{v}_{2} & \cdots & \mathbf{v}_{s}
\end{array}\right] \\
& \mathbf{y}=\left[\begin{array}{llll}
y_{1} & y_{2} & \cdots & y_{s}
\end{array}\right]^{T}
\end{aligned}
$$

For every $i=1,2, \ldots, s$, if the $i$-th selected DOF is DOF $j, \mathbf{v}_{\mathrm{i}}$ can be constructed as

$$
\mathbf{v}_{i}=\left[\begin{array}{lllllll}
0 & \cdots & 0 & 1 & 0 & \cdots & 0
\end{array}\right]^{T},\left(1 \text { is the } j \text {-th member of } \mathbf{v}_{\mathbf{i}}\right) .
$$

In this way, an independent coefficient can be determined for the selected DOF.

Substituting Eq. (12) into Eq. (11), and pre-multiplying both side by $\mathbf{r}_{B}^{T}$, Eq. (11) becomes

$$
\mathbf{r}_{B}^{T} \mathbf{K} \mathbf{r}_{B} \mathbf{y}=\mathbf{r}_{B}^{T} \boldsymbol{\delta}
$$

Defining

$$
\begin{aligned}
& \mathbf{K}_{R}=\mathbf{r}_{B}^{T} \mathbf{K} \mathbf{r}_{B} \\
& \mathbf{F}_{R}=\mathbf{r}_{B}^{T} \boldsymbol{\delta}
\end{aligned}
$$

Eq. (16) can be written as

$$
\mathbf{K}_{R} \mathbf{y}=\mathbf{F}_{R}
$$

Only Eq. (18), whose scale, the number of non-zero values, is very small needs to be solved, and $\mathbf{y}$ can be obtained. Then substituting $\mathbf{y}$ into Eq. (12), the approximation of $\Delta \mathbf{d}$ is obtained, and consequently $\mathbf{d}$ can be obtained by Eq. (6).

\subsection{Indirect Factorization Updating (IFU)}

The IFU method combined with IGA is developed from the Independent Coefficients method and the Sherman-Morrison-Woodbury (SMW) formula. Due to the fact that the structure only has local modifications, the modified equations are divided into two parts: balanced equations and unbalanced equations. Using the theory of the IC, the extra constraints are enforced on the unbalanced Degrees of Freedom (DOFs), so that the fundamental solution system of the balanced equations can be obtained by using the SMW formula. Then, a unique solution is derived from 
the general solution of the balanced equations to satisfy the unbalanced equations. In order to use the SMW formula directly, the change in the stiffness matrix are converted to a low-rank form by using the Cholesky factorization of the initial stiffness matrix. The Cholesky factorization is indirectly updated according to the stiffness matrix of the balanced equations rather than according to the modified equations.

For generality, we consider the positions of the unbalanced DOFs are arbitrary. Assuming the unbalanced DOFs are recorded in $\mathbf{S}_{d}$, and the number of unbalanced DOFs is $n_{d}$, Eq. (11) can be divided into balanced equations

$$
\mathbf{K}_{b} \Delta \mathbf{d}=\mathbf{0}
$$

and, unbalanced equations

$$
\mathbf{K}_{u} \Delta \mathbf{d}=\boldsymbol{\delta}_{u}
$$

where $\boldsymbol{\delta}_{u}$ contains the non-zero members of $\boldsymbol{\delta}, \mathbf{K}_{b}$ and $\mathbf{K}_{u}$ both have the same size as $\mathbf{K}$, but they are not simple upper and lower blocks.

Letting the displacements of the unbalanced DOFs equal to 1 , and the others equal to 0, a basis vector of the fundamental solution system of Eq. (19) can be obtained. In detail, the unbalanced DOFs need to be restrained as 0 or 1 . For the $i$-th DOF that needs to be restrained as 0 , the operation is as follows: set the $i$-th row and $i$-th column of $\mathbf{K}$ to 0 ; Then set the $i$-th diagonal member of $\mathbf{K}$ to 1; Afterwards, set the $i$-th member of the right hand vector to 0 . While, the $i$-th DOF needs to be restrained as 1 , the operation is as follows: Update right hand vector: subtract the $i$-th column of $\mathbf{K}$ from the right hand vector; Set the $i$-th row and $i$-th column of $\mathbf{K}$ to 0 ; Set the $i$-th diagonal member of $\mathbf{K}$ to 1 ; Set the $i$-th member of the right hand vector to 1. Therefore, no matter if the displacement constraint is 0 or 1 , the operation on $\mathbf{K}$ is the same. Assuming that the stiffness matrix after restraining all the unbalanced DOFs is $\mathbf{K}_{c}$, then the fundamental solution system of Eq. (19) can be obtained by solving the following linear system:

$$
\mathbf{K}_{c} \mathbf{B}=\mathbf{R}
$$

where $\mathbf{B}$ and $\mathbf{R}$ are both matrix with $n_{d}$ columns, and $\mathbf{R}$ can be calculated as follows: Firstly, for $i$ changing from 1 to $n_{d}$, we define $d=\mathbf{S}_{d}(i)$, and $\mathbf{R}_{i}=-\mathbf{K}_{d}$ (The subscript indicates a column). Secondly, for $i$ changing from 1 to $n_{d}$, we define $d=\mathbf{S}_{d}(i)$, 
$\mathbf{R}_{d}=\mathbf{0}$ (The subscript indicates a row) and $\mathbf{R}_{d, i}=1$.

Then the general solution of Eq. (19) can be calculated as

$$
\Delta \mathbf{d}=\mathbf{B y}
$$

Substituting Eq. (22) into Eq. (20), yields

$$
\mathbf{K}_{B} \mathbf{y}=\boldsymbol{\delta}_{u}
$$

where,

$$
\mathbf{K}_{B}=\mathbf{K}_{u} \mathbf{B}
$$

Solving Eq. (22), yields $\mathbf{y}$. Substituting $\mathbf{y}$ into Eq. (21), we obtain $\Delta \mathbf{d}$. Hence, the key issue is how to solve Eq. (21). Because $\mathbf{K}_{c}$ is obtained by restraining the unbalanced DOFs on $\mathbf{K}$, compared with $\mathbf{K}_{0}$ it is found that:

Condition 1: $\mathbf{K}_{c}$ equals to $\mathbf{K}_{0}$ except for the members related to the unbalanced DOFs. Therefore, restraining all the unbalanced DOFs on $\mathbf{K}$ is equivalent to restraining them on $\mathbf{K}_{0}$. Therefore, the Cholesky factorization of $\mathbf{K}_{0}$ should be considered to be reused in reanalysis. The problem now is how the restraining influences the factorization.

Assume that the $k$-th unbalanced DOF is DOF $i, \mathbf{K}_{0}$ can be assumed as

$$
\begin{aligned}
& \mathbf{K}_{0}=\left[\begin{array}{ccc}
\mathbf{K}_{a a} & \mathbf{k}_{a i} & \mathbf{K}_{a b} \\
\mathbf{k}_{i a} & k_{i i} & \mathbf{k}_{i b} \\
\mathbf{K}_{b a} & \mathbf{k}_{b i} & \mathbf{K}_{b b}
\end{array}\right]=\left[\begin{array}{ccc}
\mathbf{L}_{a a} & \mathbf{0} & \mathbf{0} \\
\mathbf{l}_{i a} & l_{i i} & \mathbf{0} \\
\mathbf{L}_{b a} & \mathbf{l}_{b i} & \mathbf{L}_{b b}
\end{array}\right]\left[\begin{array}{ccc}
\mathbf{L}_{a a}^{T} & \mathbf{l}_{i a}^{T} & \mathbf{L}_{b a}^{T} \\
\mathbf{0} & l_{i i} & \mathbf{l}_{b i}^{T} \\
\mathbf{0} & \mathbf{0} & \mathbf{L}_{b b}^{T}
\end{array}\right]=\mathbf{L L}^{T} \\
& =\left[\begin{array}{ccc}
\mathbf{L}_{a a} \mathbf{L}_{a a}^{T} & \mathbf{L}_{a a} \mathbf{l}_{i a}^{T} & \mathbf{L}_{a a} \mathbf{L}_{b a}^{T} \\
\mathbf{l}_{i a} \mathbf{L}_{a a}^{T} & \mathbf{l}_{i a} \mathbf{l}_{i a}^{T}+l_{i i} l_{i i} & \mathbf{l}_{i a} \mathbf{L}_{b a}^{T}+l_{i i} \mathbf{l}_{b i}^{T} \\
\mathbf{L}_{b a} \mathbf{L}_{a a}^{T} & \mathbf{L}_{b a} \mathbf{l}_{i a}^{T}+\mathbf{l}_{b i} l_{i i} & \mathbf{L}_{b a} \mathbf{L}_{b a}^{T}+\mathbf{l}_{b i} \mathbf{l}_{b i}^{T}+\mathbf{L}_{b b} \mathbf{L}_{b b}^{T}
\end{array}\right]
\end{aligned}
$$

After restraining, $\mathbf{K}_{0}$ becomes

$$
\mathbf{K}_{0}^{*}=\left[\begin{array}{ccc}
\mathbf{K}_{a a} & \mathbf{0} & \mathbf{K}_{a b} \\
\mathbf{0} & 1 & \mathbf{0} \\
\mathbf{K}_{b a} & \mathbf{0} & \mathbf{K}_{b b}
\end{array}\right]=\left[\begin{array}{ccc}
\mathbf{L}_{a a} \mathbf{L}_{a a}^{T} & \mathbf{0} & \mathbf{L}_{a a} \mathbf{L}_{b a}^{T} \\
\mathbf{0} & 1 & \mathbf{0} \\
\mathbf{L}_{b a} \mathbf{L}_{a a}^{T} & \mathbf{0} & \mathbf{L}_{b a} \mathbf{L}_{b a}^{T}+\mathbf{l}_{b i} \mathbf{l}_{b i}^{T}+\mathbf{L}_{b b} \mathbf{L}_{b b}^{T}
\end{array}\right]
$$

If the similar operation is applied to $\mathbf{L}$, then $\mathbf{L}$ becomes 


$$
\mathbf{L}_{0}=\left[\begin{array}{ccc}
\mathbf{L}_{a a} & \mathbf{0} & \mathbf{0} \\
\mathbf{0} & 1 & \mathbf{0} \\
\mathbf{L}_{b a} & \mathbf{0} & \mathbf{L}_{b b}
\end{array}\right]
$$

Define

$$
\mathbf{K}_{0}^{\prime}=\mathbf{L}_{0} \mathbf{L}_{0}^{T}=\left[\begin{array}{ccc}
\mathbf{L}_{a a} \mathbf{L}_{a a}^{T} & \mathbf{0} & \mathbf{L}_{a a} \mathbf{L}_{b a}^{T} \\
\mathbf{0} & 1 & \mathbf{0} \\
\mathbf{L}_{b a} \mathbf{L}_{a a}^{T} & \mathbf{0} & \mathbf{L}_{b a} \mathbf{L}_{b a}^{T}+\mathbf{L}_{b b} \mathbf{L}_{b b}^{T}
\end{array}\right]
$$

Comparing Eq. (26) and Eq. (28), it can be found that the difference only appears on the low right block.

Define

$$
\mathbf{l}_{k}=\left(\begin{array}{c}
\mathbf{0} \\
\mathbf{l}_{b i}
\end{array}\right)
$$

and,

$$
\Delta \mathbf{K}=\mathbf{l}_{k} \mathbf{l}_{k}^{T}
$$

then $\mathbf{K}_{0}^{*}$ and $\mathbf{K}_{0}^{\prime}$ have the following relationship:

$$
\mathbf{K}_{0}^{*}=\mathbf{K}_{0}^{\prime}+\Delta \mathbf{K}
$$

From Eq. (30), it is found that the modification of a single DOF can be expressed as a rank-one modification. For multiple constraints, the operation is similar. However, a specific situation should be noted as follows.

Assume that another DOF - the $j$-th DOF $(j>i)$ needs to be restrained. Since $i<j$, $\Delta \mathbf{K}_{i, j}$ may be non-zero according to Eq. (20). After restraining the $j$-th DOF, $\Delta \mathbf{K}_{i, j}$ should be zero. If we calculate $\Delta \mathbf{K}$ as

$$
\Delta \mathbf{K}=\mathbf{l}_{k} \mathbf{I}_{k}^{T}+\mathbf{l}_{k+1} \mathbf{l}_{k+1}^{T}
$$

it will lead to an error. In this situation, the $j$-th member of $\mathbf{l}_{k}$ should be set to 0 . A better way to avoid this problem is to restrain $\mathbf{L}$ from bottom to top. In this way, no extra operation on $\mathbf{l}_{k}$ is needed.

Generally, after all unbalanced DOFs are restrained on $\mathbf{K}$ and $\mathbf{L}, \mathbf{K}$ becomes $\mathbf{K}_{c}$ and $\mathbf{L}$ becomes $\mathbf{L}_{c}$. The relationship between them is

$$
\mathbf{K}_{c}=\mathbf{L}_{c} \mathbf{L}_{c}^{T}+\mathbf{l}_{1} \mathbf{l}_{1}^{T}+\mathbf{l}_{1} \mathbf{l}_{1}^{T}+\cdots+\mathbf{l}_{n_{d}} \mathbf{l}_{n_{d}}^{T}
$$

Define 


$$
\mathbf{V}=\left[\begin{array}{llll}
\mathbf{l}_{1} & \mathbf{l}_{2} & \cdots & \mathbf{l}_{n_{d}}
\end{array}\right]
$$

Eq. (33) becomes

$$
\mathbf{K}_{c}=\mathbf{L}_{c} \mathbf{L}_{c}^{T}+\mathbf{V} \mathbf{V}^{T}
$$

According to the SMW formula, Eq. (21) can be solved as

$$
\mathbf{B}=\left(\left(\mathbf{L}_{c} \mathbf{L}_{c}^{T}\right)^{-1}-\left(\mathbf{L}_{c} \mathbf{L}_{c}^{T}\right)^{-1}\left(I+\mathbf{V}\left(\mathbf{L}_{c} \mathbf{L}_{c}^{T}\right)^{-1} \mathbf{V}^{T}\right)^{-1}\left(\mathbf{L}_{c} \mathbf{L}_{c}^{T}\right)^{-1}\right) \mathbf{R}
$$

Since the SMW formula is an exact reanalysis method, the solution of the proposed method is also an exact one. From this section, it can be found that the Cholesky factorization of the initial stiffness matrix is indirectly updated according to the balanced equations, but not directly according to the modified equations. Therefore, the proposed method is termed as 'Indirect Factorization Updating'.

In addition, because equations with different stiffness matrices might yield the same solution, Condition 1 cannot be guaranteed if the unbalanced DOFs are selected by using Eq. (10) directly. A new selection criterion is suggested as follows:

$$
\Delta=\operatorname{sum}\left(\left|\mathbf{K}-\mathbf{K}_{0}\right|\right)+|\boldsymbol{\delta}|
$$

where $|\bullet|$ indicates absolute value, and $\operatorname{sum}(\bullet)$ indicates row sum.

The flowchart of the IGA- based IFU method is summarized in Fig. 5. It should be noted that, due to the fact that there are no assumptions in formulation and process of derivation, this proposed IGA- based IFU is an exact reanalysis method. It means if you modify more DOFs (increase the $n_{\mathrm{d}}$ ), only the efficiency is affected and costing more time; but the accuracy is preserved and can be guaranteed all the time regardless of how you modify the shape or boundary. Hence, the proposed IGA- based IFU will show better performance when $n_{\mathrm{d}}$ is smaller or the whole scale is larger.

Besides, another most noteworthy advantage of this proposed method is that it extends and combines IFU with IGA; technically, it has no limitation on the CAD model or how the CAD model is represented. This is due to the fact that it starts from the equilibrium equation $\mathrm{Kd}=\mathrm{F}$. That means whether the geometry is watertight or trimmed B-rep, whether the geometry that is used has unstructured or structured meshes, whether how many patches are used, whether how complicated the geometry is, once the geometry is represented in IGA and the formulation $\mathrm{Kd}=\mathrm{F}$ is obtained using IGA, this method can be used. 


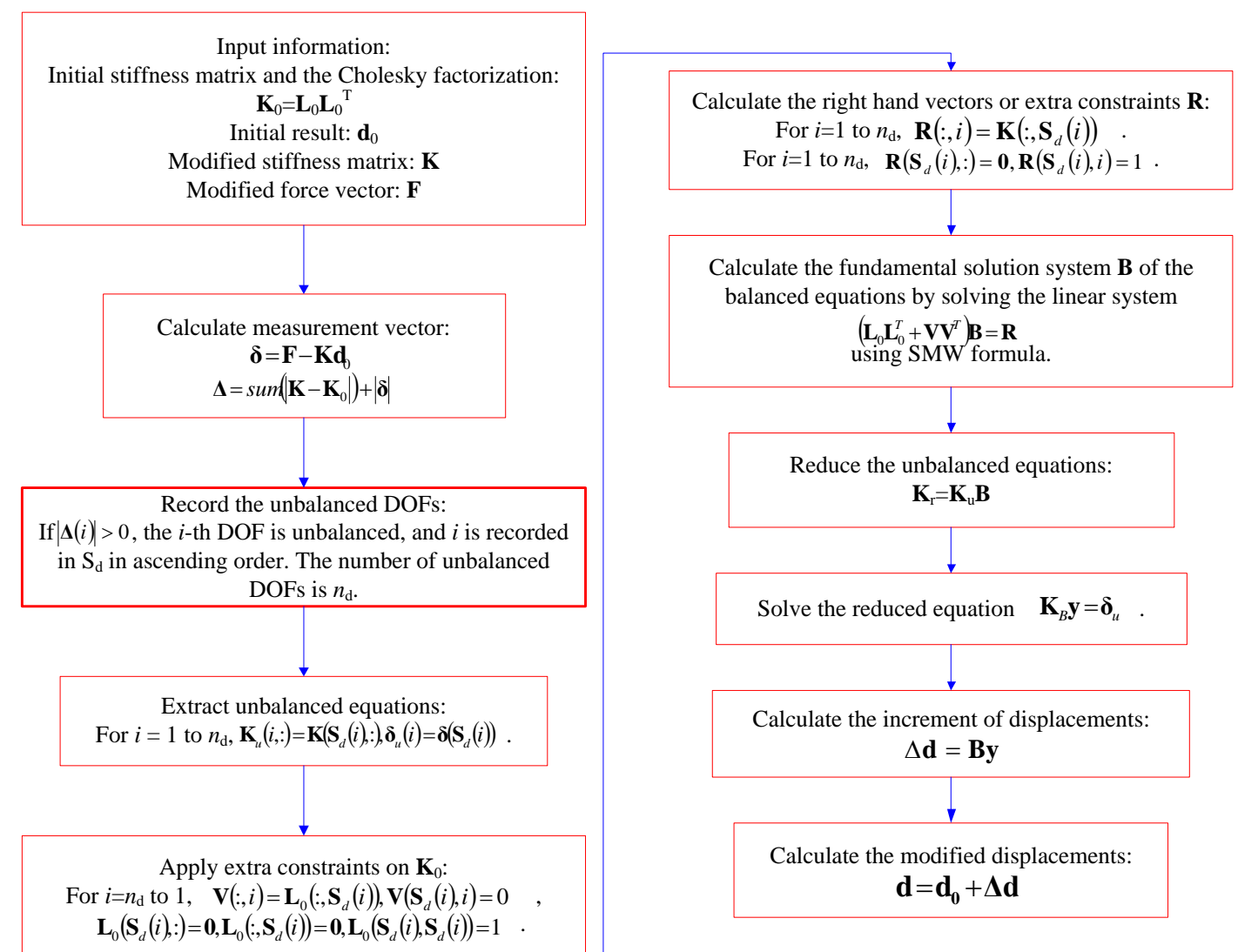

Note: If $\mathbf{A}$ is a matrix, $\mathbf{A}(i,:)$ indicatese the $i$-th row of $\mathbf{A}$, and $\mathbf{A}(:, i)$ indicates the $i$-th column of $\mathbf{A}$.

Fig. 5. Flowchart of the IFU method.

\section{Examples:}

\subsection{Modifications in structural shape}

The first example is IGA reanalysis of a blade with modifications in shape. The Blade is generally regarded as the most critical component of a turbine and turbofan. Its design requirements, such as strain, limits along the fiber direction and surface stress limit, and are specified by the design requirements. A whole lot of analysis and optimizations of the blade are needed in its design process. But its shape is impossible to be exactly represented by traditional methods, and its design and analysis processes are tedious and time-consuming; it may even fail in the end.

Hence, considering all of these factors, particularly its requirements and shape features, a blade is adopted and illustrated in Fig. 6. This blade is represented by NURBS three-dimension elements. The red nodes are control points and the elements are distinguished by a blue gird. One of its faces is fixed while all others are free. Its 
material properties are: Young's modulus $\mathrm{E}=1 \times 10^{4} \mathrm{~N} / \mathrm{cm}^{2}$ and Poisson's ratio $v=0.3$. As comparison, a complete analysis that includes constructing the modified whole stiffness matrix $\mathrm{K}$ and solving the new set of equations is conducted to verify the efficiency and accuracy of IGA reanalysis via IFU for accurate shape modification.
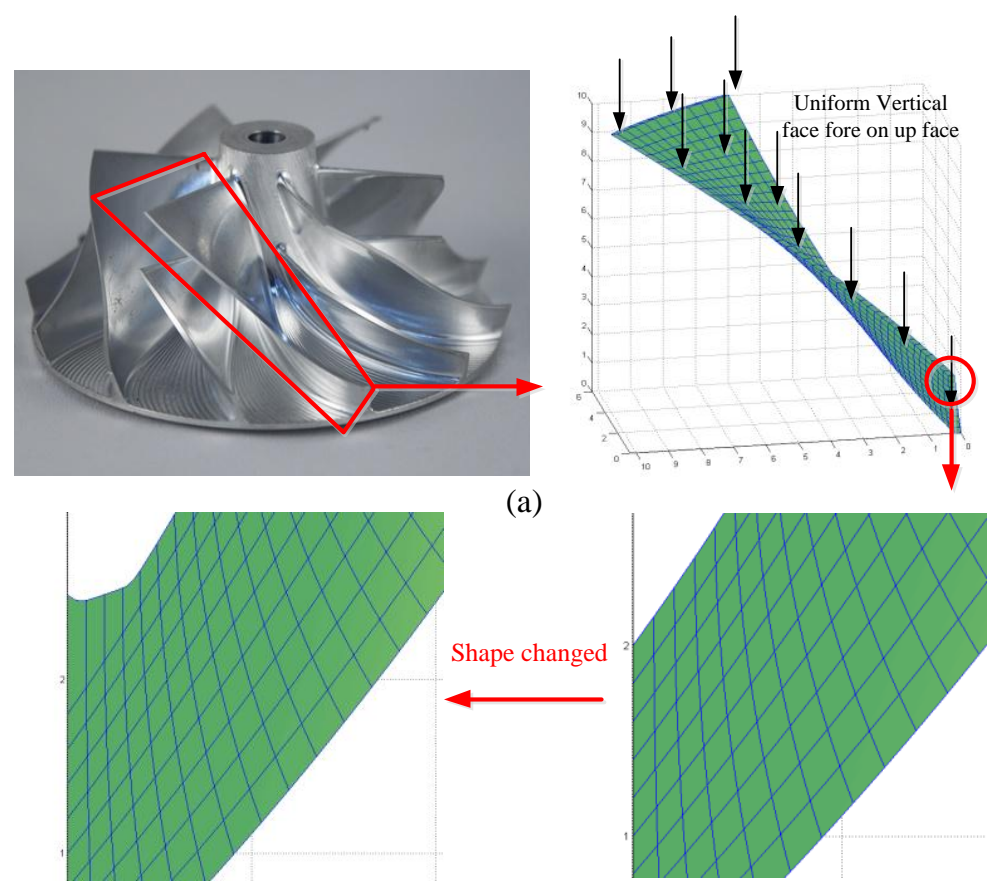

(b)

Fig. 6. (a) Blade in turbine and geometrical model presented by NURBS with force;

(b) Initial model and model with accurate shape modification.

Although all kinds of shape modifications can be made, we arbitrarily change 18 DOFs in a total of 2592 DOFs to produce the shape modification as shown in Fig. 6(b). The IGA reanalysis used the proposed IFU method and complete analysis of the structure with shape modification, and both are conducted for comparison. Besides, we refined the model several times to explore how the accuracy and efficiency of the proposed IGA reanalysis will change when the total number of DOFs changes. The relative error between displacements used in the complete analysis and IGA reanalysis -IFU is observed as indicator to verify its effectiveness. And the time costs by complete analysis and IFU are compared to verify the efficiency. 


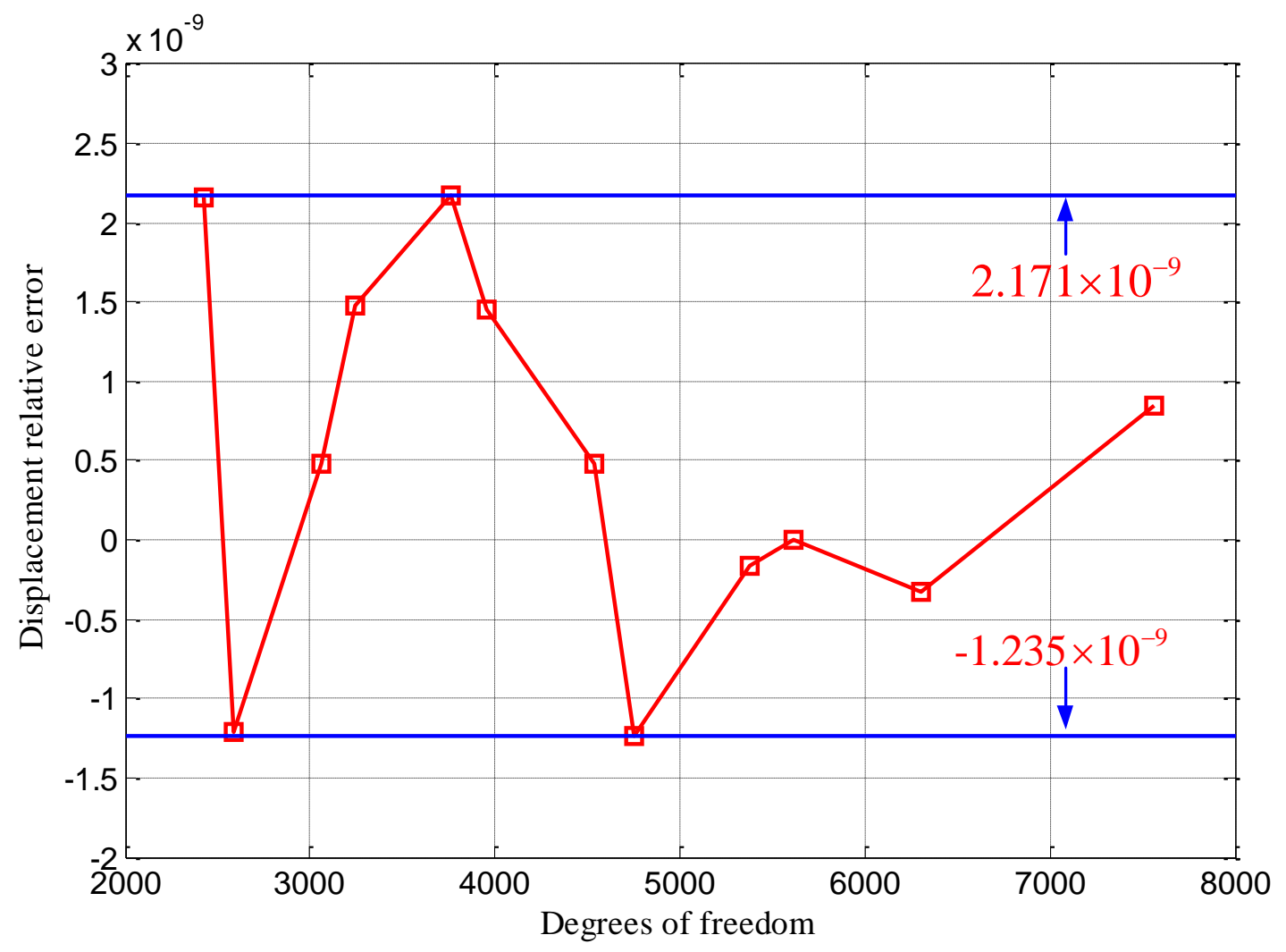

Fig. 7. Displacement relative error with DOFs.

In Fig. 7, the red line shows the relative errors between the displacements used in complete reanalysis and displacements used via IFU. From the aspect of efficiency, the blue line implies the error varies around zero from $-1.235 \times 10^{-9}$ to $2.171 \times 10^{-9}$ with increase in degrees of freedom. Furthermore, even when the scale, namely the number of total DOFs is small, the relative error reaches $10^{-9}$, which verifies that IFU is an exact reanalysis method, and almost no error is produced by adopting IGA based IFU to analyze the new structure with accurate shape modification. 


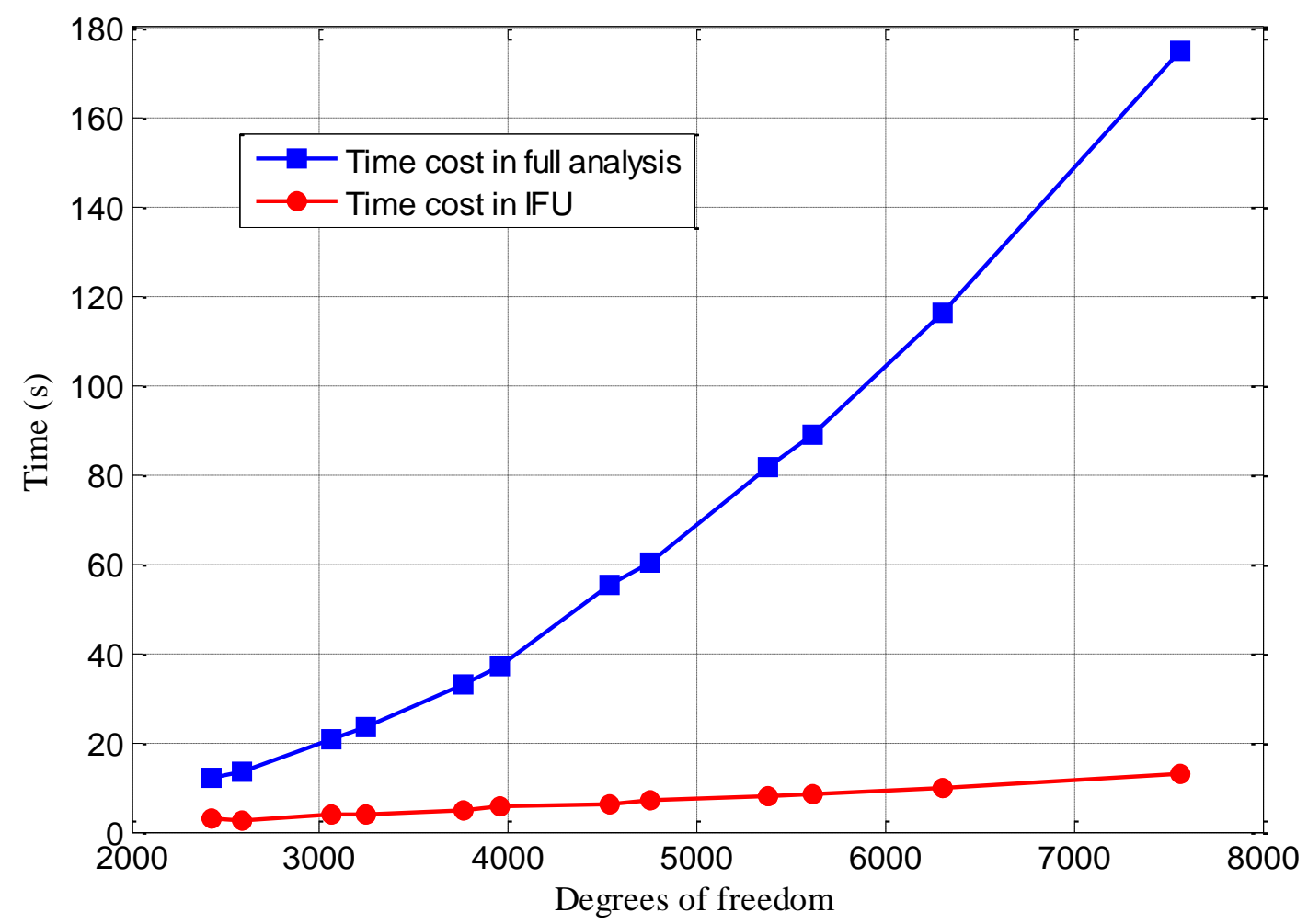

Fig. 8. Time cost in complete analysis and IFU with DOFs.

Also, looking at the Fig. 8 that shows the efficiency, the blue line with square markers shows the CPU time spent by complete analysis. We observe that when the number of DOFs increases, the cost for the CPU time increases with an accelerated speed. It means that a huge computational time is needed especially in analyzing large-scale problems. This is because the more degrees of freedom the structure has, the more time is needed to solve the equilibrium Eq. 6, especially for calculating the inverse of the modified stiffness matrix.

But for IGA reanalysis employing IFU, the cost of the CPU time represented by red line with circle markers is small, and increases slowly as the number of DOFs increases. Comparing these two lines, we can clearly observe that using IFU can result in savings of enormous CPU time in calculating structure response with accurate shape modification. The difference between cost times used by the two methods will become larger as the number of DOFs increases. The larger the problem's scale, the more efficient the proposed method will be. This implies that using IFU could save enormous calculation time, resulting in a huge significance in engineering applications. This is especially true for large-scale problems and problems which need a lot of iterative calculations, as well as providing a powerful tool and a solid foundation for future researchers. 


\subsection{Modifications in boundary conditions}

The second example is an exact reanalysis employing IFU for accurate modifications in boundary conditions. Boundary modification is a typical and most challenging kind of low-rank modification; this is because after the boundary is modified, the deformation of the structure may change dramatically. Therefore, the Scordelis-Lo roof with modifications in boundary condition is presented in this section as an illustration. As shown in Fig. 9, the length of the roof is $L=50 \mathrm{ft}$ and its radius is $R=25 f t$. The thickness is $\mathrm{t}=0.25 \mathrm{ft}$ and span angle is $\varphi=40^{\circ}$. Its material properties are Young's modulus $E=4.32 \times 10^{8} \mathrm{~N} / f t^{2}$ and Poisson's ratio $v=0.3$. The two curved edges are supported by a rigid-diaphragm. Its upper face is subjected to a vertical force per unit area of $\mathrm{g}=-90 \mathrm{~N} / \mathrm{ft}^{2}$. Similarly, a complete analysis is also conducted as comparison to verify the efficiency and accuracy of IGA reanalysis employing IFU.

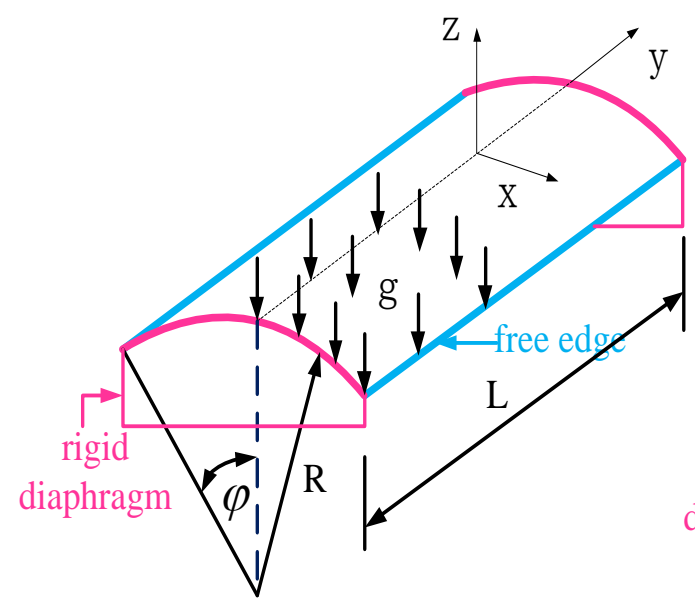

(a)

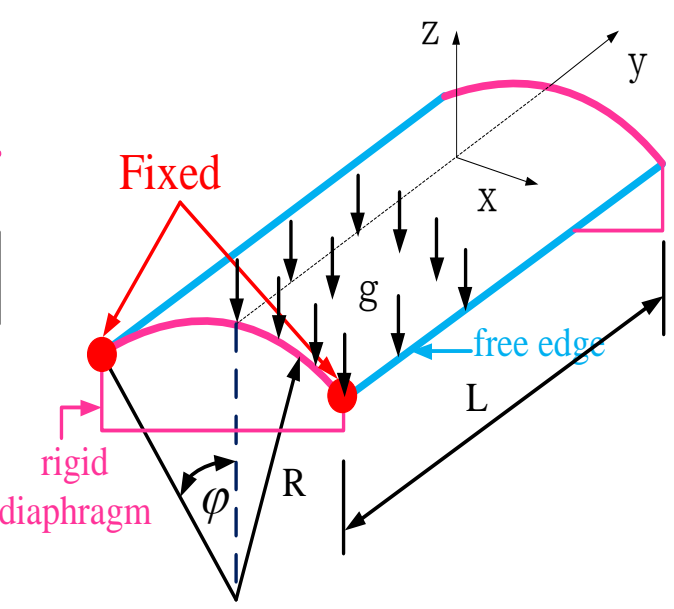

(b)

Fig. 9. (a) Original Scordelis-Lo Roof model;

(b) Scordelis-Lo Roof model with boundary modification.

In IGA reanalysis employing IFU for boundary modification, we fixed two corners as Fig. 9 (b) shows to produce boundary modification, and increased the number of fixed DOFs from 96 to 100. Then, a complete analysis and IGA reanalysis employing IFU are both conducted and compared. Besides, we refined the model several times in order to study the relationship between the accuracy and efficiency of the proposed IGA reanalysis and number of DOFs. The relative error between 
displacements used for complete analysis and displacement used via IFU is observed to prove its accuracy, and the time cost due to complete analysis and IFU to verify efficiency.

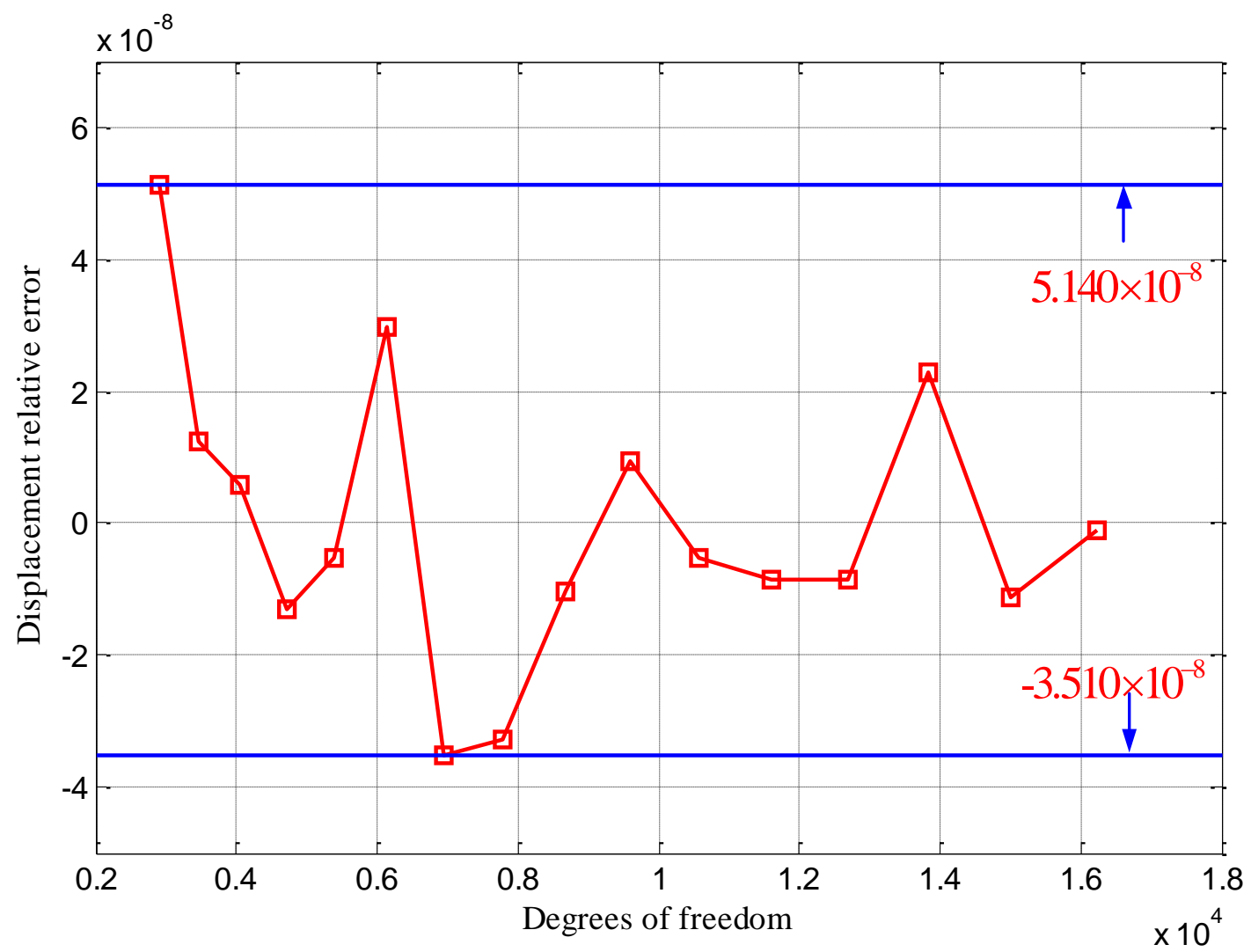

Fig. 10. Displacement relative error with DOFs.

As shown in Fig. 10, the red line depicts the relative displacement error between displacement used complete reanalysis and displacement using IFU. From this line, we can find that the error varies from $-3.510 \times 10^{-8}$ to $5.140 \times 10^{-8}$ as the number of DOFs increases. The error between the complete analysis and reanalysis using IFU is very small, and to $10^{-8}$. This verifies that IFU is an accurate reanalysis method for accurate modification in boundary. It matches the fact that there is no theoretical error between reanalysis results and full analysis results. No error is produced by adopting IGA and IFU to calculate structure with boundary modifications. 


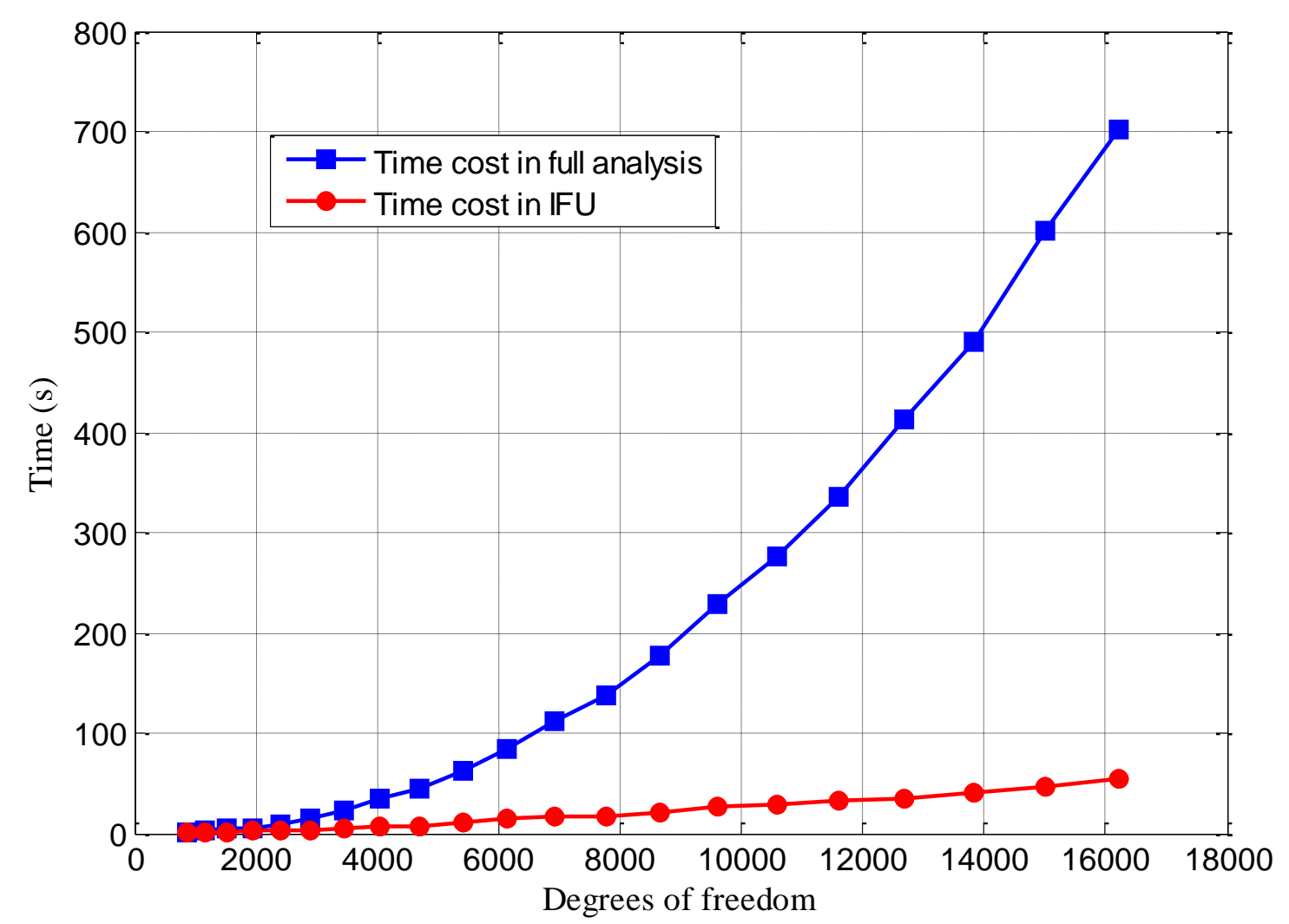

Fig. 11. Time cost in complete analysis and IFU with DOFs.

For its efficiency, as shown in Fig. 11, the blue line with square markers shows the CPU time spent in complete analysis; and the CPU time cost by using the proposed method is shown by red line with circle markers Comparing these two, we can definitely observe that, as the number of DOFs increase, the time cost by using traditional methods increases with an accelerated speed due to the fact that the larger the number of DOFs is, the more time is needed to solve the equilibrium Eq. (6). However, the CPU time spent by using the IGA based IFU reanalysis is extremely small and increases very slowly. Additionally, the difference of the CPU time will become much bigger as the number of DOFs increases. This implies that IFU is very efficient, and employing IFU will save enormous time in calculating structure response with boundary modifications. The larger the problem's scale, the higher the efficiency of IFU will be the end result, whilst providing a powerful tool and a solid foundation for future researchers.

\section{Conclusion:}

An exact and efficient novel isogeometric reanalysis of accurate modifications in shape and boundary was presented in this paper. Based upon the formulations and 
derivation and numerical examples, the following conclusions can be made:

Firstly, by using the proposed method in design and analysis, the exact geometrical model can be represented, and modifications, especially modification in shape can be accurately represented and simultaneously transformed without errors.

Secondly, this proposed method, technically, has no limitation on the CAD model or how the CAD model is represented. It is applicable to all techniques of representing CAD geometry model and complex problems.

Thirdly, the proposed IGA based IFU is an exact reanalysis method and there is no theoretical error. And due to the fact that it does not solve the complete set of modified equations, this method saves a lot of computational time, fostering analysis and improving the efficiency.

Lastly, the accuracy of the proposed method is preserved and guaranteed regardless of how many DOFs will need to be modified and/or how to modify. Furthermore, the larger scale of the problem, the more advantageous the proposed method will be.

The proposed method takes full use of the advantages of IGA in CADICAE integration and IFU exactly and efficiently in CAE reanalysis, and has huge significance in real engineering applications.

\section{Acknowledgements:}

This work was supported by the State Key Program of National Natural Science of China (61232014); National Science Foundation of China (11472101); Hunan Provincial Innovation Foundation For Postgraduate (CX2016B079)

\section{Reference}

1. P.W. Christensen, A. Klarbring, An Introduction to Structural Optimization, Springer, Netherlands, 2010.

2. K.K. Choi, N.H. Kim, Structural Sensitivity Analysis and Optimization 1: Linear Systems, Springer, New York, 2005.

3. J. Haslinger, R.A.E. Mäkinen, Introduction to Shape Optimization: Theory, Approximation, and Computation SIAM, Society for Industrial and Applied Mathematics, Philadelphia, 2003.

4. O.C. Zienkiewicz, R.L. Taylor, The Finite Element Method, fifth ed., Butterworth Heinemann, Oxford, UK, 2000.

5. V.P. Nguyena, C.Anitescuc, S.P.A.Bordasa, T. Rabczukc, Isogeometric analysis: An overview and computer implementation aspects. Mathematics and Computers 
in Simulation. 11 (2015) 789-116.

6. T.J.R. Hughes, J.A. Cottrell, Y. Bazilevs, Isogeometric analysis: CAD, finite elements, NURBS, exact geometry and mesh refinement, Computer Methods in Applied Mechanics and Engineering. 194 (2005) 4135-4195.

7. J.A. Cottrell, T.J.R. Hughes, Y. Bazilevs, Isogeometric Analysis: Toward Integration of CAD and FEA, Wiley, New York, 2009.

8. L. Piegl, W. Tiller. The NURBS book (Monographs in Visual Communication), 2nd ed. Springer, Verlag Berlin Heidelberg, 1995.

9. T.J.R. Hughes, The finite element method: linear static and dynamic finite element analysis, Prentice-Hall, Englewood Cliffs, N.J, 1987

10. W.A. Wall, M.A. Frenzel, C. Cyron, Isogeometric structural shape optimization, Computer Methods in Applied Mechanics and Engineering. 197 (2008) 29762988.

11. X.P. Qian, Full analytical sensitivities in NURBS based isogeometric shape optimization. Computer Methods in Applied Mechanics and Engineering. 199 (2010) 2059-2071.

12. A.P. Nagy, M.M. Abdalla, Z. Gurdal, Isogeometric sizing and shape optimization of beam structures. Computer Methods in Applied Mechanics and Engineering. 199 (2010) 1216-230.

13. D. Fußeder, B. Simeon, A.V. Vuong, Fundamental aspects of shape optimization in the context of isogeometric analysis, Computer Methods in Applied Mechanics and Engineering. 286 (2015) 313-331.

14. C.S. Ding, X.Y. Cui, G.Y. Li, Accurate analysis and thickness optimization of tailor rolled blanks based on isogeometric analysis. Struct Multidisc Optim. 54 (2016) 871-887

15. L. Dedè, M.J. Borden, T.J.R. Hughes, Isogeometric Analysis for Topology Optimization with a Phase Field Model, Arch Comput Methods Eng. 19 (2012) 427-465.

16. Y. Bazilevs, T.J.R. Hughes. Weak imposition of Dirichlet boundary conditions in fluid mechanics, Computers \& Fluids. 36(1) (2007) 12-26.

17. Y. Bazilevs, T.J.R. Hughes. NURBS-based isogeometric analysis for the computation of flows about rotating components, Computational Mechanics. 43(1) (2008)143-150.

18. P.N. Nielsen, A.R. Gersborg, J. Gravesen, N.L. Pedersen. Discretizations in isogeometric analysis of Navier-Stokes flow, Computer Methods in Applied Mechanics and Engineering. 200 (45-46) (2011) 3242-3253.

19. Y. Bazilevs, V.M. Calo, Y. Zhang, T.J.R. Hughes. Isogeometric fluid-structure interaction analysis with applications to arterial blood flow, Computational Mechanics. 38 (4-5) (2006) 310-322.

20. Y. Bazilevs, M.C. Hsu, M.A. Scott. Isogeometric fluid-structure interaction analysis with emphasis on non-matching discretizations and with application to wind turbines, Computer Methods in Applied Mechanics and Engineering, 249- 
252 (2012) 28-41.

21. Y. Bazilevs, C.C. Long, A.L. Marsden, Fluid-structure interaction simulation of pulsatile ventricular assist devices, Computational Mechanics, 52 (5) (2013) 971981.

22. L.D. Lorenzis, I. Temizer, P. Wriggers, G. A. Zavarise, large deformation frictional contact formulation using NURBS-based isogeometric analysis. International Journal for Numerical Methods in Engineering. 87 (13) (2011) 1278-1300.

23. I. Temizer, P. Wriggers, T.J.R. Hughes, Contact treatment in isogeometric analysis with NURBS, Computer Methods in Applied Mechanics and Engineering. 200 (9-12) (2011) 1100-1112.

24. L. Jia, Isogeometric contact analysis: geometric basis and formulation for frictionless contact, Comput. Methods Appl. Mech. Engrg. 200 (5-8) (2011) 726741.

25. C.V. Verhoosel, M.A. Scott, T.J.R. Hughes, D. R. Borst. An isogeometric analysis approach to gradient damage models, International Journal for Numerical Methods in Engineering. 86 (1) (2011) 115-134.

26. F. Irzal, J.J.C. Remmers, C.V. Verhoosel, R. Borst, An isogeometric analysis B'ezier interface element for mechanical and poromechanical fracture problems, International Journal for Numerical Methods in Engineering. 97 (8) (2014) 608628.

27. U. Kirsch (1981) Approximate structural reanalysis based on series expansion. Comput Methods Appl Mech Eng 26(2):205-223

28. U. Kirsch, Combined approximations - a general reanalysis approach for structural optimization, Struct. Multidisc. Optim. 20 (2000) 97-106.

29. U. Kirsch, P.Y. Papalambros, Structural reanalysis for topological modifications a unified approach, Struct. Multidisc. Optim. 21 (2001) 333-344.

30. U. Kirsch, A unified reanalysis approach for structural analysis, design, and optimization. Struct, Multidisc. Optim. 25 (2003) 67-85.

31. U. Kirsch, P.Y. Papalambros, Exact and accurate reanalysis of structures for geometrical changes, Engineering with computers. 17 (2001) 363-372.

32. F. Rong, S. Chen, Y. Chen, Structural modal reanalysis for topological modifications with extended Kirsch method, Comput. Methods Appl. Mech. Engng. 192 (2003) 697-707.

33. U. Kirsch, M. Bogomolni, I. Sheinman, Nonlinear dynamic reanalysis of structures by combined approximations, Comput. Methods Appl. Engng. 195 (2006) 4420-4432.

34. G. Gao, H. Wang, G. Li, An adaptive time-based global method for dynamic reanalysis, Struct. Multidisc. Optim. 48 (2013) 355-365.

35. G. Zhang, E. Nikolaidis, Z.P. Mourelatos, An efficient re-analysis methodology for probabilistic vibration of large-scale structures, Journal of Mechanical Design. 131 (2009) 1-13. 
36. S. Chen, Z. Yang, A universal method for structural static reanalysis of topological modification, Int. J. Numer. Meth. Engng. 61 (2004) 673-686.

37. Z. Yang, X. Chen, R. Kelly, An adaptive static reanalysis method for structural modifications using epsilon algorithm, Computational Sciences and Optimization: Theory, Simulation and Experiment. 2 (2009) 897-899.

38. S. Chen, L. Ma, G. Meng, Dynamic response reanalysis for modified structures under arbitrary excitation using epsilon-algorithm, Computers and Structures. 86 (2008) 2095-2101.

39. U. Kirsch, M. Kocvara, J. Zowe, Accurate reanalysis of structures by a preconditioned conjugate gradient method, Int. J. Numer. Meth. Engng. 55 (2002) 233-251.

40. B.S. Wu, C. Lim, Z. Li, A finite element algorithm for reanalysis of structures with added degrees of freedom, Finite Elements in Analysis and Design. 40 (2004) 1791-1801.

41. B.S. Wu, Z. Li, Reanalysis of structural modifications due to removal of degrees of freedom, Acta Mechanica. 180 (2005) 61-71.

42. B.S. Wu, Z. Li, Static reanalysis of Structures with added degrees of freedom, Commun. Numer. Meth. Engng. 22 (2006) 269-281.

43. X. Yang, S. Chen, B. Wu, Eigenvalue reanalysis of structures using perturbations and Padé approximation, Mechanical Systems and Signal Processing. 15 (2) (2001) 257-263.

44. X. Yang, H. Lian, S. Chen, An adaptive iteration algorithm for structural modal reanalysis of topological modifications, Commun. Numer. Meth. Engng. 18 (2002) 373-382.

45. B.S. Wu, Z. Li, S. Li, The implementation of a vector-value rational approximate method in structural reanalysis problems, Comput. Methods Appl. Mech. Engng. 192 (2003) 1773-1784.

46. J. Sherman, W.J. Morrison, Adjustment of an inverse matrix corresponding to changes in the elements of a given column or a given row of the original matrix, Ann. Math. Stat. 20 (4) (1949) 621.

47. M. Woodbury. Inverting modified matrices, Memorandum Report, 42. Statistical Research Group. Princeton University, Princeton, 1950.

48. L.S. Tuckeman, Divergence-free velocity fields in nonperiodic geometries, Journal of Computational Physics. 80 (1989) 403-441.

49. T.A. Davis, W.W. Hager, Modifying a sparse Cholesky factorization, SIAM J. MATRIX ANAL. APPL. 20 (1999) 606-627.

50. T.A. Davis, W.W. Hager, Multiple-rank modifications of a sparse Cholesky factorization. SIAM J, MATRIX ANAL. APPL. 22 (2001) 997-1013.

51. H.F. Liu, B.S. Wu, Z.G. Li, Method of updating the Cholesky factorization for structural reanalysis with added degrees of freedom, Journal of Engineering Mechanics. 140 (2) (2013) 384-392.

52. H.F. Liu, B.S. Wu, Z.G. Li, S. P. Zheng, Structural static reanalysis for 
modification of supports, Struct. Multidisc. Optim. 50 (2014) 425-435.

53. Q. Song, P. Chen, S. Sun, An exact reanalysis algorithm for local non-topological high-rank structural modifications in finite element analysis, Computers and Structures. 143 (2014) 60-72.

54. M. Cheikh, A. Loredo, Static reanalysis of discrete elastic structures with reflexive inverse, Applied Mathematical Modelling. 26 (2002) 877-891.

55. G.X. Huang, H. Wang, G.Y. Li, A reanalysis method for local modification and the application in large-scale problems. Struct. Multidisc. Optim. 49 (2014) 915930.

56. G.X. Huang, H. Wang, G.Y. Li, An exact reanalysis method for structures with local modifications. Struct. Multidisc. Optim. 54 (2016) 499-509.

57. G.Q. Gao, H. Wang, E.Y. Li, G.Y. Li. An exact block-based reanalysis method for local modifications. Computers and Structures. 158 (2015) 369-380.

58. B.H.V. Topping, A.M. A. Kassim, Theorems of geometric variation for finite element analysis. Int J Numer Methods Eng. 26 (1988) 2577-606

59. A.M. AKassim, B.H.V. Topping, The theorems of geometric variation for nonlinear finite element analysis. Comput Struct. 25 (1987) 877-93

60. A. Kaveh, H. Fazli, Approximate eigensolution of locally modified regular structures using a substructuring technique. Comput Struct. 89 (2011) 529-37.

61. A. Kaveh, H. Fazli, M.A. Asl, S.R. Mirghaderi, Analysis of regular structures with member irregularity using the equilibrium equations and the singular value decomposition. Adv Struct Eng. 16 (2013) 823-43.

62. H. Rahami, A. Kaveh, I. Shojaei, Y. Gholipour, Analysis of irregular structures composed of regular and irregular parts using graph products. J Comput Civ Eng. 28 (2014) 